\title{
Backstory of "Molcas 8: New capabilities for multiconfigurational quantum chemical calculations across the periodic table"
}

\author{
Roland Lindh ${ }^{1}$ and Josh Nicholson ${ }^{2}$ \\ ${ }^{1}$ Backstories \\ ${ }^{2}$ Authorea Team
}

October 21, 2020

The subject paper(Aquilante et al., 2015) is the 5th paper in a series of papers(Aquilante et al., 2012; Roos et al., 1990, 1991; Veryazov et al., 2004; Aquilante et al., 2010) on the development of the MOLCAS program package. In this short back story I will try to put the MOLCAS quantum chemistry program package into a brief historical context, shortly describe its development, and finally argue the case why papers like the subject paper is needed.

The Molcas project started in 1989 by the theoretical chemistry group of the late Prof. Björn Roos (see Figure 1) at Lund University, Sweden. The Swedish government struck a deal with the banks - no increase of tax if they supported research. As a consequence of this the Molcas project materialised as a collaboration between IBM and the research group in Lund. Swedish theoretical chemistry had made a serious impart on the ab inition field at the time with contributions from researchers as Jan Almlöf, Per. E. M. Siegbahn, and Björn Roos - the former two the first Ph.D. students of the latter at Stockholm University. During this time the three of them had developed specialized software. Jan Almöf developed the Molecule(Taylor, 2017) program (computation of two-electron integrals), Per E. M. Siegbahn the MRCI code(Siegbahn, 1992; Roos and Siegbahn, 1977) (multi reference configuration interaction), and Björn O. Roos developed the CASSCF program(Roos et al., 1980) (complete active space self-consistent field). The goal of the Molcas project was to bring these pieces of software together in a single package designed for the IBM 3090 machine. Version 1.0 was distributed to the public in the late 1989. Subsequent versions were released 1991, 1993, 1997, 2000, 2003, 2007, and 2014, covering version 2-8. All versions have been commercial versions.

Today the package support multiple options and methods, and several hardware and software platforms. In 2005 the project started the "Molcas users' workshops" with the most recent workshop, the 8th, taking place in Uppsala November 2017. Over the time and under the leadership of Björn Roos the project have had several success stories which have been seminal to the field. Let us mention two here, the complete active space 2 nd order perturbation theory model(Andersson and Roos, 1993) and the complete active space state interaction(Malmqvist and Roos, 1989) method. From the formation of the project until about 2010 the project was mainly a project which was heavenly dominated by the Lund group, especially with respect to the leadership and strategic decisions, however, with significant programming contributions from international collaborators. During 2009 Björn Roos retired from the project due to poor health(Siegbahn and lindh, 2011), the baton was passed on to the long time Molcas co-developer Roland Lindh. Starting in Zürich 2013 the first "Molcas developers' workshop" took place. This has been followed by annual workshops at Alcalá (Spain), Siena (Italy), Vienna (Austria), Jerusalem (Israel) and this year at Leuven (Belgium). During the same time the project have developed from a national Swedish project - dominated by a single Swedish research group - to an international project with 30-40 active developers from some 10 different universities 
and institutes. The authors list of the subject paper is a testament to this development. In 2017 the project went open-source having the most significant part being released under the "Lesser GLP" license and is now distributed free of charge under the name of OpenMolcas.

The subject paper was written on the request of the developers after one of our developers' workshops. People argued that a single paper, including the most recent developments, would be needed to make new developments and implementations known to the computational chemistry community. Additionally, the issue of lack of recognition and credits for software development was mentioned as one of the most important reasons for the need of a paper like the subject paper - in many aspects a mini-review paper with no novel contributions. Normally hard-working software developers seldom get proper credits for their work, although it can and is fundamental to the ability to perform accurate quantum chemical simulations. In particular if this development is not associated with new wave-function models. Some of us, like me, contribute with significant software and methods, which are completely instrumental for the calculations, but hardly ever get any credit for this contribution. Let me give an example, I'll use my own contribution, two-electron integrals (Lindh et al., 1991) (since long also a part of MOLPRO), which without no calculations with the package would be possible, as an example. Since its publication in 1991 this paper, on the computation of two-electron integrals, has, according to Google Scholar, attracted 258 citations. In the same time the two packages have attracted 7249 citation - the use of the two-electron code was surely significant to the research the citations corresponds to but they handed credit to the developer in less than $3.6 \%$ of the time. If I would have designed the basis set, however, I would have been assured the full 7249 citations - we always cited the basis sets but hardly ever how we efficiently compute the matrix elements they generate. There are several other developments and features in a quantum chemistry package which are not considered worthy citations but are still as essential to calculations. Here comes the paper, as the subject paper, in as an equalizer and makes sure that all developers of a package gets the credit and respect they deserve. With these type of papers around we kill two flies with one stone - we reduce the number of references to theoretical papers and at the same time make sure that all developers get the recognition they all deserve and need. 


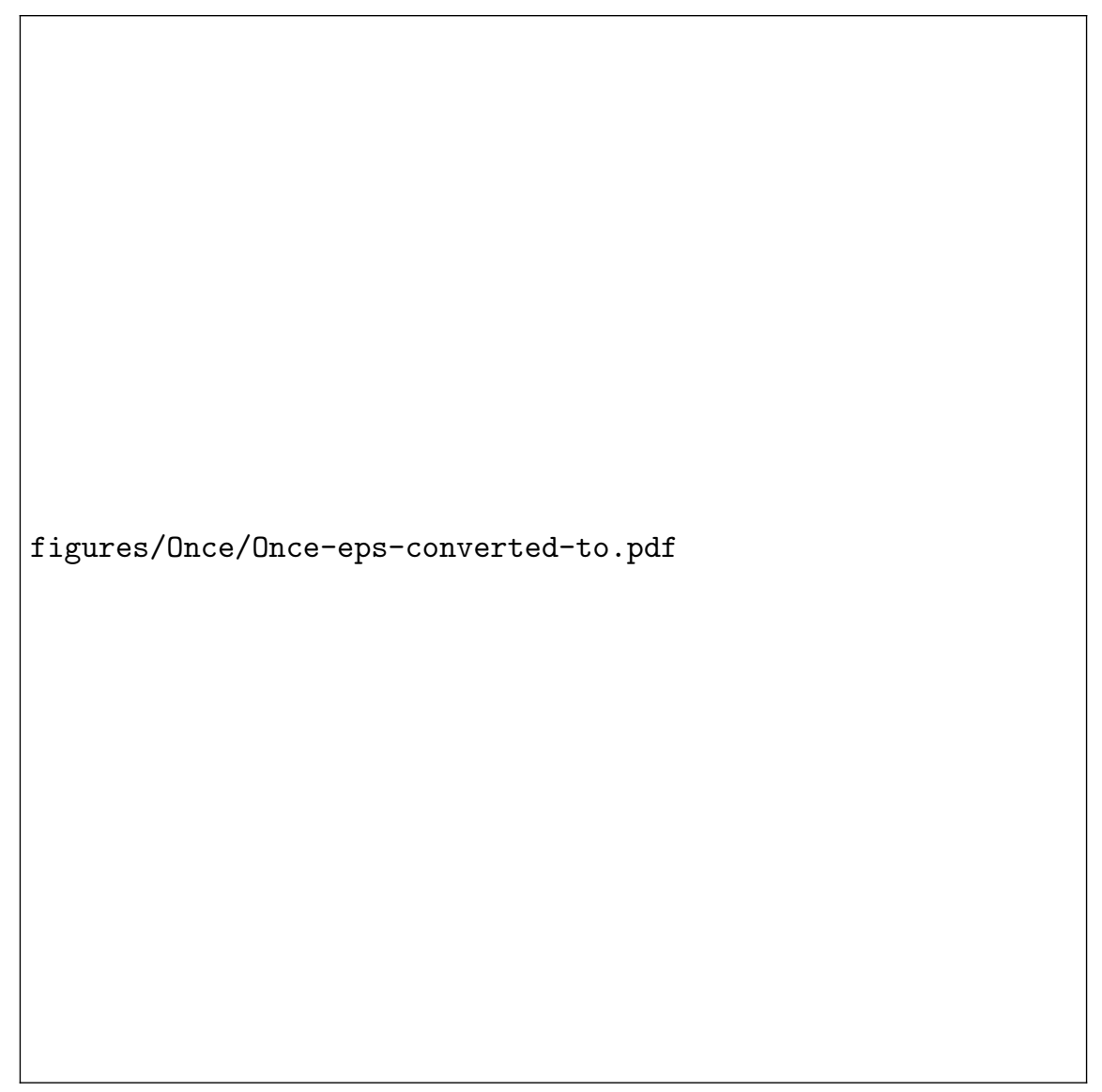

Figure 1: Björn O. Roos standing next to the so-called BESK computer in Stockholm. The picture is from sometime during the earl1960ties.

\section{References}

Kerstin Andersson and Björn O. Roos. Multiconfigurational second-order perturbation theory: A test of geometries and binding energies. International Journal of Quantum Chemistry, 45(6):591-607, 1993. doi: 10.1002/qua.560450610. URL https://doi.org/10.1002\%2Fqua. 560450610.

Francesco Aquilante, Luca De Vico, Nicolas Ferré, Giovanni Ghigo, Per Åke Malmqvist, Pavel Neogrády, Thomas Bondo Pedersen, Michal Pitonák, Markus Reiher, Björn O. Roos, Luis Serrano-Andés, Miroslav Urban, Valera Veryazov, and Roland Lindh. MOLCAS 7: The Next Generation. Journal of Computational Chemistry, 31(1):224-247, jan 2010. doi: 10.1002/jcc.21318. URL https://doi.org/10.1002\%2Fjcc. 21318.

Francesco Aquilante, Thomas Bondo Pedersen, Valera Veryazov, and Roland Lindh. MOLCAS-a software for multiconfigurational quantum chemistry calculations. Wiley Interdisciplinary Reviews: Computational Molecular Science, 3(2):143-149, sep 2012. doi: 10.1002/wcms.1117. URL https://doi.org/10.1002\% 2Fwcms. 1117.

Francesco Aquilante, Jochen Autschbach, Rebecca K. Carlson, Liviu F. Chibotaru, Mickaël G. Delcey, Luca De Vico, Ignacio Fdez. Galván, Nicolas Ferré, Luis Manuel Frutos, Laura Gagliardi, Marco Garavelli, Angelo Giussani, Chad E. Hoyer, Giovanni Li Manni, Hans Lischka, Dongxia Ma, Per Åke Malmqvist, Thomas Müller, Artur Nenov, Massimo Olivucci, Thomas Bondo Pedersen, Daoling Peng, Felix Plasser, 
Ben Pritchard, Markus Reiher, Ivan Rivalta, Igor Schapiro, Javier Segarra-Martí, Michael Stenrup, Donald G. Truhlar, Liviu Ungur, Alessio Valentini, Steven Vancoillie, Valera Veryazov, Victor P. Vysotskiy, Oliver Weingart, Felipe Zapata, and Roland Lindh. Molcas8: New capabilities for multiconfigurational quantum chemical calculations across the periodic table. Journal of Computational Chemistry, 37(5): 506-541, nov 2015. doi: 10.1002/jcc.24221. URL https://doi.org/10.1002\%2Fjcc. 24221.

R. Lindh, U. Ryu, and B. Liu. The reduced multiplication scheme of the Rys quadrature and new recurrence relations for auxiliary function based two-electron integral evaluation. The Journal of Chemical Physics, 95(8):5889-5897, oct 1991. doi: 10.1063/1.461610. URL https://doi.org/10.1063\%2F1.461610.

Per-Åke Malmqvist and Björn O. Roos. The CASSCF state interaction method. Chemical Physics Letters, 155(2):189-194, feb 1989. doi: 10.1016/0009-2614(89)85347-3. URL https://doi .org/10.1016\%2F0009$2614 \% 2889 \% 2985347-3$.

B. O. Roos, G. Karlström, P.-Å. Malmqvist, A. J. Sadlej, and P.-O. Widmark. MOLCAS: A General Purpose Quantum Chemistry Program System for Correlated Wavefunctions. In Modern Techniques in Computational Chemistry: MOTECC ${ }^{T M}-90$, pages 533-552. Springer Netherlands, 1990. doi: 10.1007/97894-009-2219-8_9. URL https://doi.org/10.1007\%2F978-94-009-2219-8_9.

B. O. Roos, G. Karlström, P. Å. Malmqvist, A. J. Sadlej, and P. O. Widmark. MOLCAS: A General Purpose Quantum Chemistry Program System for Correlated Wavefunctions. In Modem Techniques in Computational Chemistry: MOTECC-91, pages 435-454. Springer Netherlands, 1991. doi: 10.1007/97894-011-3032-5_10. URL https://doi.org/10.1007\%2F978-94-011-3032-5_10.

Björn O. Roos and Per E. M. Siegbahn. The Direct Configuration Interaction Method from Molecular Integrals. In Methods of Electronic Structure Theory, pages 277-318. Springer US, 1977. doi: 10.1007/9781-4757-0887-5_7. URL https://doi.org/10.1007\%2F978-1-4757-0887-5_7.

Björn O. Roos, Peter R. Taylor, and Per E.M. Si $=$ gbahn. A complete active space SCF method (CASSCF) using a density matrix formulated super-CI approach. Chemical Physics, 48(2):157-173, may 1980. doi: 10.1016/0301-0104(80)80045-0. URL https://doi.org/10.1016\%2F0301-0104\%2880\%2980045-0.

Per Siegbahn and Roland lindh. Björn O. Roos: 1937-2010 mentor colleague, innovator. International Journal of Quantum Chemistry, 111(13):3256-3259, apr 2011. doi: 10.1002/qua.23104. URL https: //doi.org/10.1002\%2Fqua.23104.

Per E. M. Siegbahn. The Configuration Interaction Method. In Lecture Notes in Quantum Chemistry, pages 255-293. Springer Berlin Heidelberg, 1992. doi: 10.1007/978-3-642-58150-2_5. URL https://doi.org/ 10.1007\%2F978-3-642-58150-2_5.

Peter R. Taylor. Reflections on the MOLECULE integral program (and its developer). Molecular Physics, 115(17-18):2043-2047, feb 2017. doi: 10.1080/00268976.2017.1290844. URL https://doi.org/10.1080\% 2F00268976.2017.1290844.

Valera Veryazov, Per-Olof Widmark, Luis Serrano-Andrés, Roland Lindh, and Björn O. Roos. 2MOLCAS as a development platform for quantum chemistry software. International Journal of Quantum Chemistry, 100(4):626-635, 2004. doi: 10.1002/qua.20166. URL https://doi.org/10.1002\%2Fqua. 20166. 